\title{
Optic Nerve Sheath Meningocele: A Case Report
}

\author{
E. Halimi ${ }^{1}$, O. Wavreille ${ }^{1}$, R. Rosenberg ${ }^{1}$, I. Bouacha ${ }^{1}$, J.-P. Lejeune ${ }^{2}$, \\ and S. Defoort-Dhellemmes ${ }^{1}$ \\ ${ }^{1}$ Service des Explorations de la Vision et Neuro-Opthalmologie, Hôpital Roger Salengro, CHRU, Lille, France \\ and ${ }^{2}$ Service de Neurochirurgie, Hôpital Roger Salengro, CHRU, Lille, France
}

\begin{abstract}
Isolated optic nerve sheath meningocele is a rare affection defined as the cystic enlargement of the optic nerve sheath filled with cerebrospinal fluid. We report the case of a 39-year-old woman presenting with bilateral meningocele uncovered during a routine examination for headache complaints. A 5-year follow-up validated the lesion's clinical and imaging stability. Magnetic resonance imaging (MRI) is an essential tool in the diagnosis of this pathology, alongside characteristic symptoms indicating that the meningocele might have progressively expanded into the orbit. In this case we present a therapeutic approach based on pathophysiological hypotheses and review of the literature.
\end{abstract}

Keywords: Optic nerve, optic nerve sheath meningocele, tear of the optic nerve sheath

\section{OBSERVATION}

We report here the case of a 39-year-old woman who initially consulted for nonspecific headaches progressing over the past 10 years. The associated clinical symptoms included pain around the orbit and intermittent eyelid oedema.

The initial ophthalmological examination found retained visual acuity in both eyes and exophthalmos on the right side at $22 \mathrm{~mm}$ (Hertel exophthalmometer), the left side measures were normal at $19 \mathrm{~mm}$. During the cover test, intraocular pressure and oculomotricity were normal for both eyes. The examination of the anterior segment was eventless on both sides. Ophthalmoscopy of the right eye unveiled deeply located optic disc drusen validated by fluoroscopy with a cobalt filter, B-mode ultrasound, and angiography. The clinical picture brought up a pseudopapilloedema (Figure 1). The examination of the left eye was normal (Figure 2).

On the right side, visual field testing in static (Humphrey-Zeiss field analyser) and kinetic perimetry (workhorse Goldman perimeter) showed a widening of the blind spot. The latencies of evoked visual potentials (EVPs) were normal but their amplitudes diminished on both sides. B-mode ultrasound showed a larger cystic dilatation of the optic nerve sheath (Figure 3) on the right side than on the left one. Brain magnetic resonance imaging (MRI) validated an optic nerve sheath meningocele (Figure 4A, B, and C), with normal ventricles, fluid collection in enlarged subarachnoid spaces, and Grade 1 exophthalmos of the right eye (Cabanis scale).

The patient refused a lumbar puncture to check her intracranial pressure. Since the usual analgesics failed to provide pain relief for the headaches, a medical treatment with acetazolamide (half a 250-mg tablet, two to three times a day) was initiated. Pain was properly managed with acetazolamide and the meningocele remained stable during the entire 5-year follow-up, validated by yearly ophthalmology, neurosurgery, as well as MRI examinations.

\section{DISCUSSION}

The first description of "optic nerve sheath meningocele" was reported by Garrity et al. in 1990. 


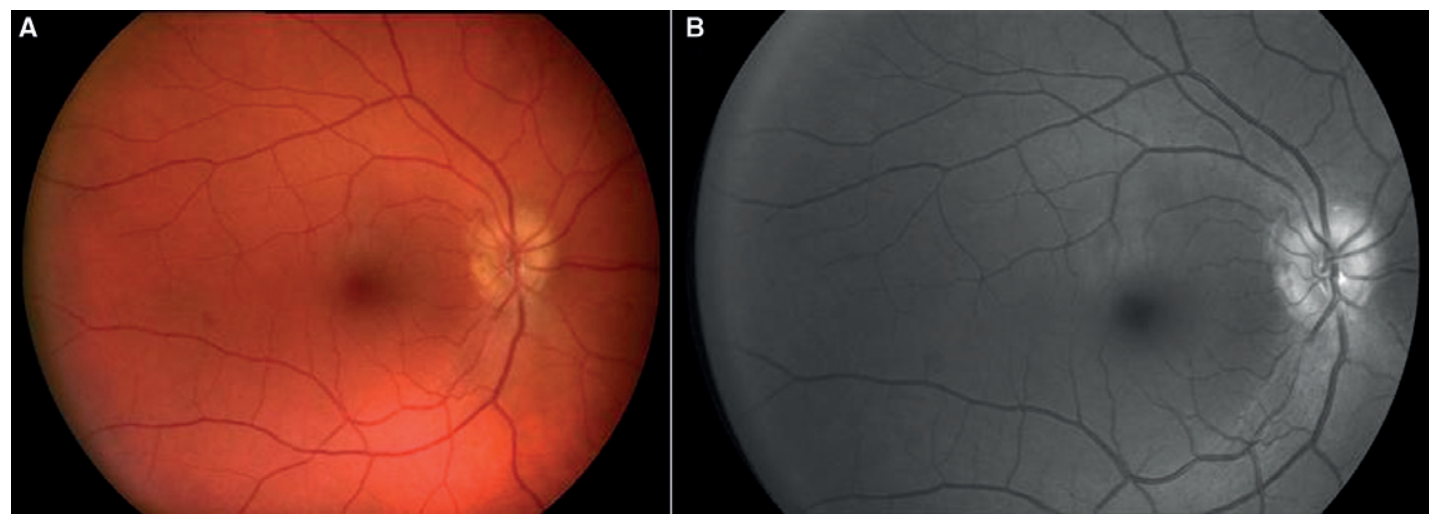

FIGURE 1. Ophthalmoscopy of the right eye. (A) Colour view. (B) Blue cobalt filter. Pseudopapilloedema of the right eye related to buried optic nerve head drusen.

Note: Figure 1 of this article is available in color online at www.informahealthcare.com/oph.

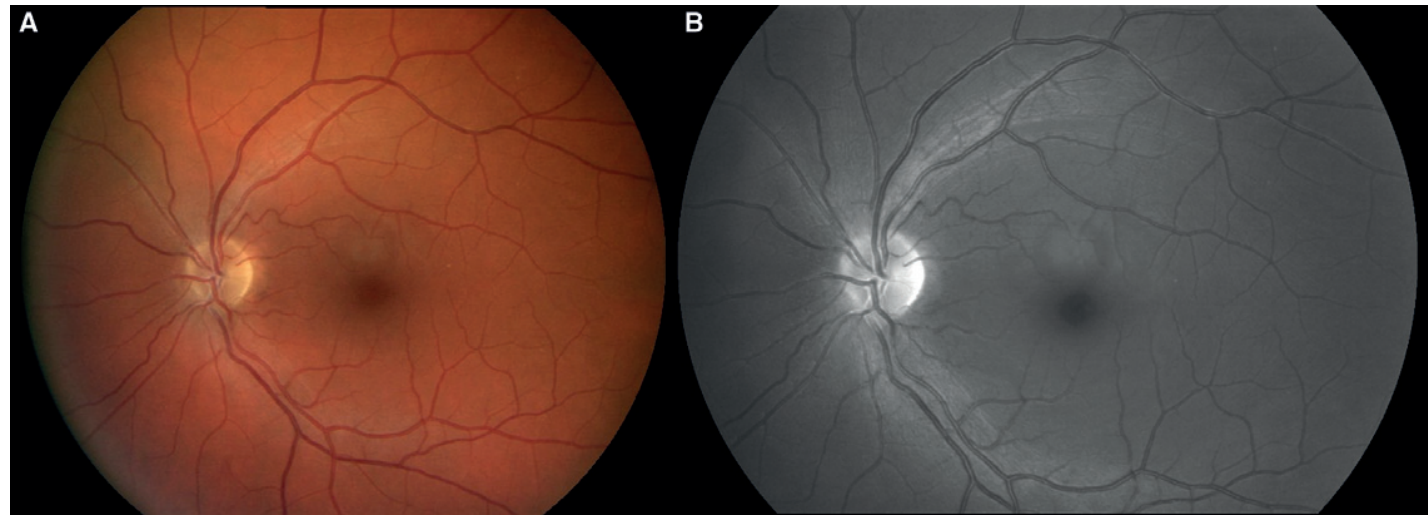

FIGURE 2. Ophthalmoscopy of the left eye. (A) Colour view. (B) Blue cobalt filter. Normal. Note: Figure 2 of this article is available in color online at www.informahealthcare.com/oph.

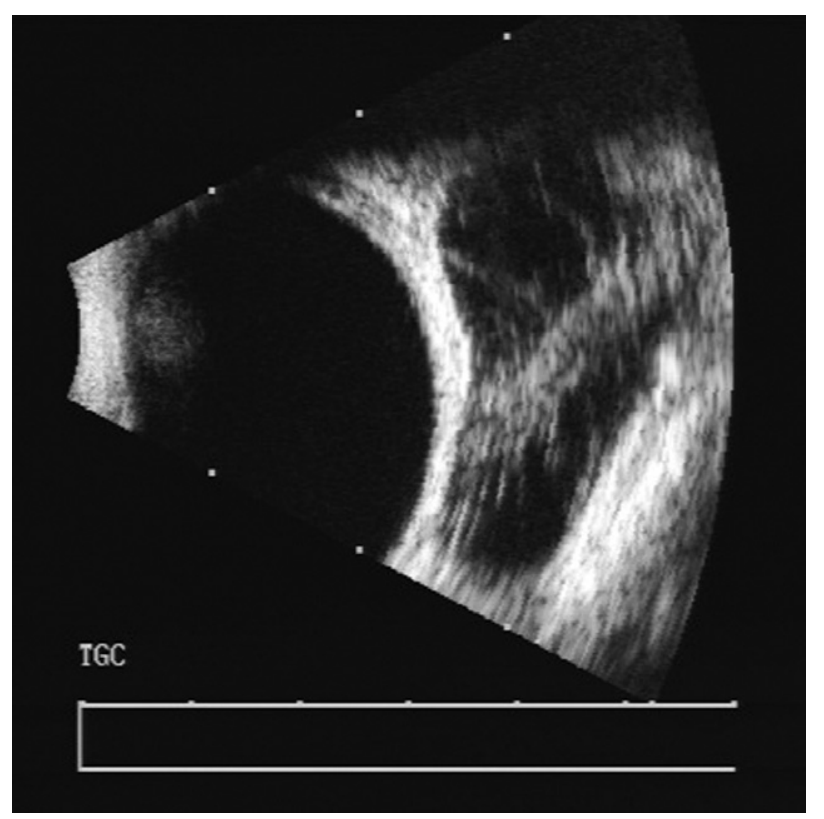

FIGURE 3. Mode-B ultrasound. Cystic dilatation of the optic nerve sheath.

This lesion was defined as cystic enlargement of the subarachnoid spaces of the optic nerve, which itself remained normal. This dilation is bilateral in most cases. Isolated optic nerve sheath meningocele is a rare affection and only about 30 cases were reported in the literature. ${ }^{2,3}$

The pathophysiology of meningocele remains unknown. ${ }^{2,4}$ Hayreh [5] reported that the subarachnoid space was narrower in the optic canal. In fact, congenital narrowing of the bony part of the optic canal and congenital abnormalities of the cranial/ orbital fusion could be the cause of meningocele by altering the cerebrospinal fluid (CSF) flow within the periorbital subarachnoid space. ${ }^{1,4,5}$ Some authors reported cases of meningocele with abnormalities in intracranial pressure and brought forward the hypothesis that its pathophysiology could be similar to idiopathic intracranial hypertension (IIH). ${ }^{3}$

Initials symptoms can be neurological and/or ophthalmological but remain vague. ${ }^{1-4}$ Neurological symptoms usually consist in nonspecific headaches. Ophthalmological symptoms can reflect a progressive expansion of the meningocele causing orbital pain, acquired hyperopia, and unilateral or bilateral exophthalmos. But some symptoms will point to complications such as acute or progressive decrease in visual acuity or alteration of the visual field. ${ }^{1-4}$

The main differential diagnoses are optic nerve glioma, meningioma (primary or secondary), and 

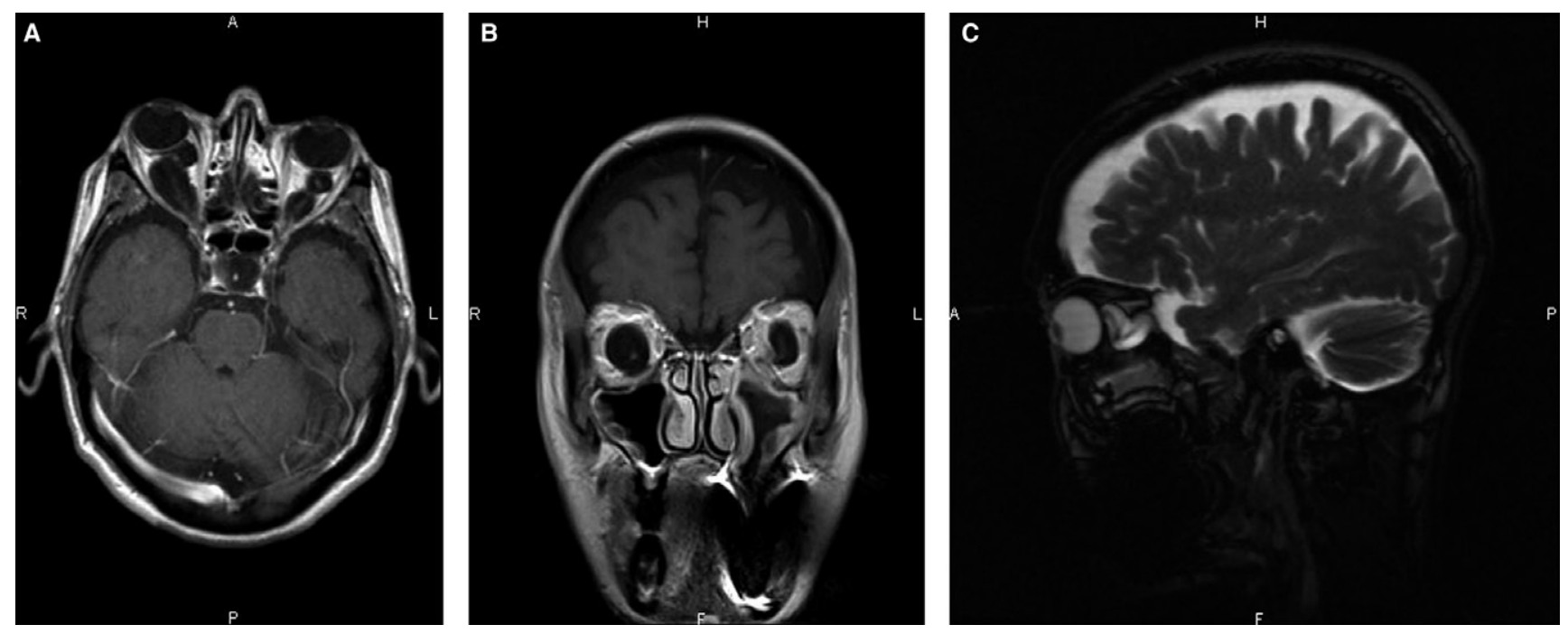

FIGURE 4. MRI of the brain and orbits. (A) Axial view, T2-FLAIR-weighted sequences. Cystic dilatation with low intensity signal (A and B) or hypersignal (C) of the optic nerve sheath and inside a normal-sized optic nerve in isosignal. Grade 1 Exophthalmos on the right side (Cabanis scale). Enlarged pericerebral fluid collection spaces.

arachnoid cysts. ${ }^{1,2,4}$ MRI is an essential element to validate the diagnosis. ${ }^{1,2,4,6}$ T1-weighted sequences show low signal intensity with normal-sized optic nerves in dilated sheaths filled with CSF, whereas T2weighted sequences unveil a hypersignal with normal-sized optic nerves in dilated sheaths filled with CSF. Fat-suppression MRI techniques are the most informative to discard intraorbital tissue lesion or compression of the optic nerve. ${ }^{1,2}$

Ultrasound imaging can be useful, since it is readily available, as well as time- and cost-effective, but it has major limits, i.e., its results depend on the quality of the operating technician and there is no direct examination of the optic canal.

Very few cases were described in the literature and no consensus seems to be emerging. If the lesion can remain stable for several years, it can also progress to acute or progressive loss of vision due to irreversible optic nerve damages. ${ }^{1}$ This is why regular ophthalmological monitoring seems essential. There are no guidelines either on the therapeutic strategy; several authors advocate close monitoring with yearly multidisciplinary check-ups. When headaches are resistant to classic analgesics or in presence of papilloedema, acetazolamide treatment should be implemented. ${ }^{4,7}$ The latter only has a positive impact on the headaches but not on the meningocele itself. Secondarily, if the papilloedema is not improved by treatment or when symptoms worsen (decrease in visual acuity, visual field alteration, exophthalmos), a surgical treatment can be proposed. 1,7,8 This treatment consists of creating an opening into the optic nerve sheath. ${ }^{1,2,4}$ This procedure is effective on headaches but does not always improve visual acuity or visual field disorders. ${ }^{1}$ Furthermore, various authors report a $30 \%$ postoperative complication rate (oculomotor disorders, obstruction of the retina central artery, and more rarely acute angle-closure glaucoma, hyphaema, and retinal ischaemia ${ }^{8}$ ).

\section{CONCLUSION}

Isolated optic nerve sheath meningocele is a rare affection with bilateral findings in most cases. The clinical picture can be neurological and/or ophthalmological but no symptom is pathognomonic of the disease. Orbital MRI is the best imaging examination to validate the diagnosis. Its evolution is poorly known. It can be stable over time, but can also lead to irreversible damages to the visual functions.

Despite the lack of consensus, several authors agree on one therapeutic strategy with a multidisciplinary annual follow-up, medical treatment by acetazolamide for papilloedema or headaches resisting to conventional treatment, and finally surgery in case of resistance or failure of the first-line medical treatment.

\section{DECLARATION OF INTEREST}

The authors report no conflicts of interest. The authors alone are responsible for the content and writing of the paper.

\section{REFERENCES}

[1] Garrity JA, Trautmann JC, Bartley GB, Forbes G, Bullock JD, Jones Jr TW, Waller RR. Optic nerve sheath meningoceles. Clinical and radiographic features in 13 
cases with a review of the literature. Ophthalmology 1990;97:1519-1531.

[2] Lunardi P, Farah JO, Ruggeri A, Nardacci B, Ferrante L, Puzzilli F. Surgically verified case of optic sheath nerve meningocele: case report with review of the literature. Neurosurg Rev 1997;20:201-205.

[3] Shanmuganathan V, Leatherbarrow B, Ansons A, Laitt R. Bilateral idopathic optic nerve sheath meningocele associated with unilateral transient cystoid macular oedema. Eye (Lond) 2002;16:800-802.

[4] Mesa-Gutiérrez JC, Quiñones SM, Ginebreda JA. Optic nerve sheath meningocele. Clin Ophthalmol 2008;2:661-668.
[5] Hayreh SS. The sheath of the optic nerve. Ophthalmologica 1984;189:54-63.

[6] Weber AL, Caruso P, Sabates NR. The optic nerve: radiologic, clinical, and pathologic evaluation. Neuroimaging Clin N Am 2005;15:175-201.

[7] Bruce BB, Biousse V, Newman NJ. Update on idiopathic intracranial hypertension. Am J Ophthalmol 2011; 152:163-169.

[8] Villain MA, Candon E, Arnaud B, Hamard H, Adenis J-P. [Optic nerve sheath decompression in optic neuropathy complicating idiopathic intracranial hypertension: a new focus]. J Fr Ophtalmol 2003;26:191-197. 